\title{
Don't stress over it
}

Our materials
replicate the
stress-strain
behaviour
nearly
completely, all
the way from
the onset of
deformation
to sample
rupture

Engineering synthetic materials that mimic the complex mechanical behaviour of biological tissues is a key challenge in tissue engineering and regenerative medicine. Sergei Sheiko and colleagues now report in Nature a rational design platform that enables the fine-tuning of tissue-like mechanical properties using synthetic elastomers.

Biological tissues adopt an astonishing variety of precisely orchestrated mechanical properties, ranging from soft and elastic, as in arteries or lung, to stiff and fragile, as in bone and cartilage. However, most synthetic materials that are used

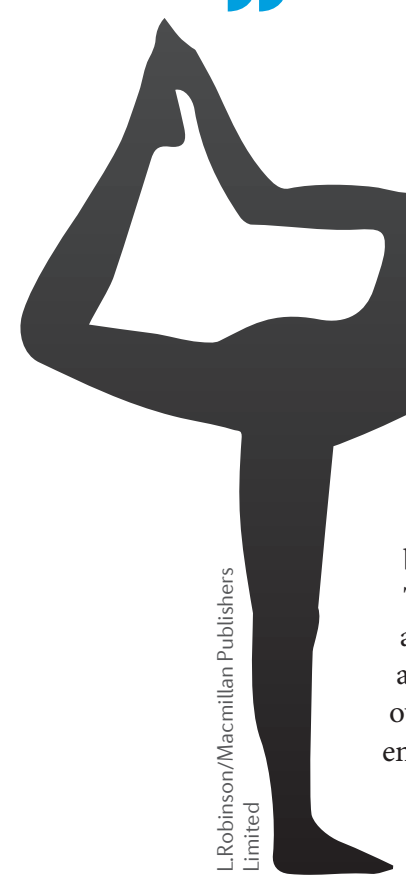
for biological applications display only a small and stringent subset of mechanical and therefore fail to produce the complex mechanical performance and stress-strain behaviour of biological tissues. This mismatch has long been a major hurdle for the clinical application of tissue mimetics owing to a lack of functional engraftment.

One of the key issues is that for linear polymers, a larger value of the elastic modulus leads to a lower value of extensibility, which hinders the synthesis of a hard but flexible material. Current tissue-mimetic technologies are mainly based on hydrogels mixtures of assorted polymers and water - that have the limitation of usually being synthesized by trial and error rather than through exact and accurate design.

Sheiko and co-workers have developed a library of brush-like polymers that are characterized by sets of three independent parameters: network strand length, side-chain length and grafting density. This library allows for the design of many combinations of mechanical properties, in particular Young's modulus, elongation-at-break and strain stiffening, and therefore enables a broad range of stress-strain behaviours.

The use of brush-like polymers also allows for the independent control of stiffness and elongation and, therefore, attributes, facilitates the creation of polymer \section{networks that mimic complex tis-} sue-like mechanics. "Current tissue mimics replicate the mechanical response of biological tissues only partially, for example, only in the initial stretch," explains Sheiko. "Our materials replicate the stress-strain behaviour nearly completely, all the way from the onset of deformation to sample rupture."

Following this strategy, the authors created mechanical replicas of strain-adaptive tissues, such as lung and arteries, using triblock co-polymers of bottlebrush poly(dimethylsiloxane) (PDMS) and linear poly(methyl methacrylate) (PMMA). Importantly, these synthetic replicas do not contain solvent, which is crucial for biomedical applications to prevent an inflammatory response. "A synthetic replica of nearly any biological tissue can be prepared on demand in a personalized manner," comments Sheiko. "If a medical doctor gives us a sample of a specific tissue, we will blueprint a polymer network architecture that will replicate the mechanical properties of this tissue precisely and accurately."

The researchers now envisage extending this technology to biocompatible and biodegradable polymers. "The impact will be great, because the range of biocompatible monomers is very narrow," concludes Sheiko. "Our 'design-by-architecture' approach will significantly expand the range of possible properties for a given biocompatible polymer." However, the road is still long for complex tissues, such as the brain. An advanced platform is currently being developed, which resolves these issues by adding chemical composition and composite structure as new variables that provide more options for property control.

Christine-Maria Horejs

ORIGINAL ARTICLE Vatankhah-Varnosfaderani, M. et al. Mimicking biological stress-strain behaviour with synthetic elastomers. Nature http://dx.doi. org/10.1038/nature23673 (2017) 